\title{
Pigment dispersion syndrome: a clinical study
}

\author{
HAROLD G. SCHEIE ${ }^{1}$ AND J. DOUGLAS CAMERON ${ }^{2}$ \\ From the ${ }^{1}$ Department of Ophthalmology, University of Pennsylvania School of Medicine, and the \\ Scheie Eye Institute, 51 North 39th Street, Philadelphia, Pennsylvania, USA, and ${ }^{2}$ Department of \\ Ophthalmology, University of Minnesota, Minneapolis, Minnesota, USA
}

SUMMARY This study involved a group of 407 patients (799 eyes) with pigment dispersion syndrome gathered from a glaucoma population of 9200 patients. The sex distribution was equal. The majority $(65 \%)$ of patients were myopic. The incidence of retinal detachment was $6.4 \%$. No patients were black, but 5 were mulatto. Approximately one-quarter of the patients with pigment dispersion syndrome ( $31 \%$ of the men, $19 \%$ of the women) had glaucoma. The average age of onset of glaucoma was 15 years less than in control patients with chronic simple glaucoma. When both eyes were affected by glaucoma, the glaucoma was consistently more severe in the eye with the more heavily pigmented angle. The degree of iris transillumination was found to be of no importance in predicting the presence of glaucoma or the severity of trabecular pigmentation. The pressure in $66 \%$ of the eyes with pigmentary glaucoma was controlled medically. A higher percentage of patients with pigmentary glaucoma required surgery than patients in the control group with chronic simple glaucoma. Men with pigmentary glaucoma required surgery at a much earlier age than women with pigmentary glaucoma.

This paper presents a statistical analysis of 799 eyes (407 patients) with pigment dispersion syndrome. Attempts were made to determine the importance of pigment as a cause of glaucoma and the response of the glaucoma to various therapeutic agents.

Pigment dispersion syndrome results from idiopathic atrophy of the pigment layers of the iris with liberation of pigment, which is carried forward and deposited along the routes of aqueous flow. Iris atrophy may result in characteristic transillumination of the iris near its base.

Pigment is usually present on the posterior capsule of the lens at the site of insertion of the posterior zonular fibres into the ligamentum hyaloideum capsulare. It may be minimal and punctate or dense and confluent in a single or double ring. The zonular fibres may have a bronzelike appearance. ${ }^{1}$ Pigment on the posterior surface of the cornea takes the form of a Krukenberg spindle, with additional punctate or dust-like deposits frequently present on the peripheral corneal endothelium and on the surface of an otherwise normal appearing iris stroma. Pigmentation of the posterior trabeculum is consistently quite dense.

Correspondence to Harold G. Scheie, MD, Scheie Eye Institute, 51 North 39th Street, Philadelphia, Pennsylvania 19104, USA.
The majority of involved eyes are myopic. Glaucoma with open angles, when it occurs, is nearly always bilateral.

\section{Materials and methods}

Four hundred and seven patients with pigment dispersion syndrome were selected from 9200 patients seen in the private practice of one of us (H.G.S.) during the years 1946 to 1977. Some had been observed for as long as 30 years. The diagnosis of pigment dispersion syndrome was dependent on the presence of a Krukenberg spindle and a heavily pigmented trabeculum. Initial evaluation of patients included visual acuity, visual fields, a complete eye examination with tonometry, and Koeppe lens gonioscopy. Water drinking tests were usually done one or more times.

The data were summarised according to (1) demographic and historical factors, (2) ocular findings, and (3) response to therapy, eyes having pigment dispersion glaucoma (pigmentary glaucoma) being compared with a control group having chronic simple glaucoma.

Seventy-two (143 eyes) patients with chronic simple glaucoma were randomly selected from the patients examined between 1961 and 1967 in the glaucoma files of one of us (H.G.S.). The selection 
technique allowed for a follow-up period of at least 10 years. No patients with pigment dispersal were included in this group.

The following criteria for the diagnosis of glaucoma, either chronic simple or associated with pigment dispersion syndrome, were arbitrarily established. The criteria were met by 143 eyes (72 patients) with chronic simple glaucoma and by 204 eyes (104 patients) with pigmentary glaucoma. (1) Intraocular pressure measurements were repeatedly $20 \mathrm{mmHg}$ or higher with characteristic cupping of the optic disc. (2) A rise of 2 scale units (Schitøz) during a water drinking test was considered confirmative. (3) Eyes with trabecular pigmentation were excluded if they had had previous ocular disease, injury or ocular surgery.

\section{Results}

\section{PIGMENT DISPERSION SYNDROME}

Age and sex

The age and sex of 407 patients (799 eyes) are shown in Table 1.

Pigment dispersion (overall group). Four hundred and seven patients (799 eyes) were included. Two hundred and four $(50 \%)$ were men and $203(50 \%)$ were women. The average age of diagnosis was $46 \cdot 3$ years for men and 49.8 years for women, but the median age at diagnosis was 44 years for men and 54 years for women, a difference of ten years.

Pigment dispersion (normotensive). Two hundred and fifty-six patients (501 eyes) showed no evidence of glaucoma. One hundred and eight $(42.2 \%)$ were men and $148(57.8 \%)$ were women with a man: woman ratio of $0 \cdot 73$. The average age at diagnosis for men and women was nearly the same ( 47.6 years and 49.6 years, respectively), but the median age was 44 years for men and 53 years for women, a difference of 9 years. Ten patients $(4.0 \%)$ gave a history of some family member with glaucoma.

Pigment dispersion (with increased pressure). Forty-seven patients (94 eyes) had increased intraocular pressure (greater than $24 \mathrm{mmHg}$ Schiøtz) without characteristic optic disc changes or visual field defects. Thirty-one $(66 \%)$ were men and 16 $(34 \%)$ were women with a man:woman ratio of 1.94 . The average age at diagnosis for men was 44.6 years and for women was 53.5 years, a difference of 8.9 years. The median age for men (42 years) was 12 years earlier than women (54 years). Nine patients $(19 \%)$ gave a history of some family member with glaucoma.

Pigment dispersion (with glaucoma). One hundred and four patients (204 eyes) had glaucoma. Sixty-five $(62.5 \%)$ were men and $39(37.5 \%)$ were women, with a man:woman ratio of 1.66 . The average age at
Table 1 Sex and age distribution

\begin{tabular}{|c|c|c|}
\hline & Men & Women \\
\hline \multicolumn{3}{|c|}{ Pigment dispersion (overall group) } \\
\hline Patients & $204(50 \%)$ & $203(50 \%)$ \\
\hline Average age & 46.3 years & 49.8 years \\
\hline Median age & 44 years & 54 years \\
\hline Age range & 14 to 84 years & 19 to 74 years \\
\hline \multicolumn{3}{|c|}{ Pigment dispersion (normotensive) } \\
\hline Patients & $108(42 \cdot 2 \%)$ & $148(57 \cdot 8 \%)$ \\
\hline Average age & $47 \cdot 6$ years & $49 \cdot 6$ years \\
\hline Median age & 44 years & 53 years \\
\hline Age range & 14 to 85 years & 19 to 75 years \\
\hline \multicolumn{3}{|c|}{$\begin{array}{l}\text { Pigment dispersion } \\
\text { (increased pressure) }\end{array}$} \\
\hline Patients & $31(66 \%)$ & $16(34 \%)$ \\
\hline Average age & $44 \cdot 6$ years & 53.5 years \\
\hline Median age & 42 years & 54 years \\
\hline Age range & 25 to 72 years & 44 to 68 years \\
\hline \multicolumn{3}{|c|}{ Pigment dispersion (glaucoma) } \\
\hline Patients & $65(62 \cdot 5 \%)$ & $39(37 \cdot 5 \%)$ \\
\hline Average age & $45 \cdot 8$ years & $50 \cdot 2$ years \\
\hline Median age & 46 years & 53 years \\
\hline Age range & 18 to 84 years & 21 to 77 years \\
\hline
\end{tabular}

diagnosis was 45.8 years for men and 50.2 years for women, a difference of 4 years. The median age was 46 years for men and 53 years for women, a difference of 7 years.

Familial pigmentary glaucoma was encountered only once. This occurred in a mulatto family in which 5 members of two generations were affected. Pigment dispersion syndrome was seen in no black patient. Eleven $(16 \%)$ of the patients with pigmentary glaucoma gave a history of another family member with glaucoma, type unknown.

\section{Colour of iris}

The colour of the iris (Table 2) was recorded in 558 eyes. The distribution of the iris colour within the 3 groups was nearly the same. In the normotensive group $171(49.3 \%)$ had brown irides, $119(34.2 \%)$ had blue, and $57(16.5 \%)$ had hazel. In the group with increased pressure $38(59.4 \%)$ had brown irides, $25(39.1 \%)$ had blue, and $1(1.5 \%)$ had hazel. In the group with glaucoma $72(49.0 \%)$ had brown irides, while $50(34.0 \%)$ had blue and $25(17.0 \%)$ had hazel.

\section{Transillumination of iris}

The irides in over half $(57.9 \%)$ of the eyes in the pigment dispersion group with normal pressure showed some degree of characteristic transcleral, basal iris transillumination (Table 2). Classified as maximal (circumferential) were $32 \cdot 1 \%$. Figures were similar in the increased pressure group, with $53 \%$ showing some transillumination and $39.5 \%$ described 
Table 2 Iris colour and transillumination

\begin{tabular}{|c|c|c|c|}
\hline & $\begin{array}{l}\text { Non-glaucoma } \\
\text { group }\end{array}$ & $\begin{array}{l}\text { Increased } \\
\text { pressure }\end{array}$ & Glaucoma \\
\hline \multicolumn{4}{|l|}{ Brown eyes } \\
\hline No transillumination & $116(68 \%)$ & $26(68 \%)$ & $50(69 \%)$ \\
\hline Localised & $12(7 \%)$ & $1(2 \%)$ & $5(7 \%)$ \\
\hline Minimal & $3(2 \%)$ & $2(4 \%)$ & $0(0 \%)$ \\
\hline Moderate & $11(6 \%)$ & $2(4 \%)$ & $3(4 \%)$ \\
\hline Maximal & $29(17 \%)$ & $7(22 \%)$ & $14(20 \%)$ \\
\hline Total & $171(100 \%)$ & $38(100 \%)$ & $72(100 \%)$ \\
\hline \multicolumn{4}{|l|}{ Blue eyes } \\
\hline No transillumination & $28(24 \%)$ & $9(36 \%)$ & $12(24 \%)$ \\
\hline Localised & $16(13 \%)$ & $1(4 \%)$ & $5(10 \%)$ \\
\hline Minimal & $0(0 \%)$ & $0(0 \%)$ & $0(0 \%)$ \\
\hline Moderate & $23(19 \%)$ & $5(25 \%)$ & $11(22 \%)$ \\
\hline Maximal & $52(44 \%)$ & $10(28 \%)$ & $22(44 \%)$ \\
\hline Total & $119(100 \%)$ & $25(100 \%)$ & $50(100 \%)$ \\
\hline \multicolumn{4}{|l|}{ Hazel eyes } \\
\hline No transillumination & $25(44 \%)$ & $1(100 \%)$ & $2(8 \%)$ \\
\hline Localised & $10(18 \%)$ & $0(0 \%)$ & $0(0 \%)$ \\
\hline Minimal & $0(0 \%)$ & $0(0 \%)$ & $0(0 \%)$ \\
\hline Moderate & $7(12 \%)$ & $0(0 \%)$ & $2(8 \%)$ \\
\hline Maximal & $15(26 \%)$ & $0(0 \%)$ & $21(84 \%)$ \\
\hline Total & $57(100 \%)$ & $1(100 \%)$ & $25(100 \%)$ \\
\hline
\end{tabular}

as maximal, and in the glaucoma group with $64.3 \%$ having transillumination $39.4 \%$ of which were maximal.

Data on transillumination should always include a statement of colour of the iris. Transillumination has a much higher frequency in blue eyes than in brown because of the paucity of stromal pigment. Approximately $75 \%$ of the blue eyes and $32 \%$ of the brown eyes in all groups showed transillumination, with very little difference among the groups for each iris colour. Transillumination therefore was found to be of no importance in predicting the presence of glaucoma or severity of trabecular pigmentation.

Gonioscopic appearance of anterior chamber angle The anterior chamber angles of all eyes reported in this paper were open. Most of the eyes with or without pigmentary glaucoma $(85.1 \%)$ showed heavy pigmentation of the trabecula (grade III or grade IV2). The incidence of embryonic tissue in the angles was not high.

A different degree of trabecular pigmentation in the two eyes was present in $38(36.5 \%)$ patients with glaucoma. The glaucoma was more severe in the eye with the more heavily pigmented trabeculum in 18 of these patients. Ten of the patients with unequal pigmentation required surgery to control the pressure in the more pigmented eye, whereas the pressure was controlled medically in the fellow eye.

Refractive error

Myopia greater than $-1.00 \mathrm{D}$ was the refractive error of 493 eyes $(62 \%)$ in the overall pigment dispersion group. Hyperopia of greater than $+1.00 \mathrm{D}$ was found in 103 eyes $(13 \%)$. The remaining 199 eyes $(25 \%)$ lay between $-0.75 \mathrm{D}$ and $+0.75 \mathrm{D}$. There was no significant difference among the 3 groups of patients.

\section{Associated ophthalmic abnormalities}

Retinal detachment occurred in $6.4 \%$ of all the patients in the study.

Eighteen eyes (17 patients, $6.6 \%$ ) in the pigment dispersion group without glaucoma had retinal detachments. Ten were men $(58.8 \%)$ with an average age of 53 years and 7 were women $(41.2 \%)$, average age 48.6 years, when diagnosed. Thirteen of the eyes were phakic and two were aphakic. Two of the detachments followed trauma (both eyes were phakic). Two eyes were $-6.00 \mathrm{D}$ spherical equivalent and 2 eyes were $-14.00 \mathrm{D}$ spherical equivalent. Of the remaining eyes 10 were myopic, 2 emmetropic, and 1 hyperopic.

One retinal detachment relating to childhood trauma occurred in the group with pigment dispersion and increased pressure.

In the pigmentary glaucoma group 9 eyes $(8$ patients, $7.6 \%$ ) had retinal detachments, and 5 eyes (5 patients) had other retinal lesions. Seven were men and 1 was a woman. The average age at diagnosis was 44 years. The remainder were all phakic, and 1 patient (with bilateral detachments) was quite myopic $(-7.00 \mathrm{D})$.

Other coexisting abnormalities in the pigment dispersion group included 11 patients with retinal disorders other than detachment, 13 patients with corneal lesions, including 6 with Fuchs's endothelial dystrophy and 1 with bilateral megalocornea, 6 patients with various type of ocular inflammatory disease, and 1 patient with congenital nystagmus.

In the pigmentary glaucoma group 2 patients had uveitis and 1 had subluxated lenses.

\section{CHRONIC SIMPLE GLAUCOMA}

CONTROL GROUP

The control group of eyes with chronic simple glaucoma consisted of 143 eyes (72 patients).

\section{INCIDENCE}

In the overall population of 9200 glaucoma patients from which the eyes in this study were selected only $15 \%$ were estimated to have chronic simple glaucoma.

Age, sex, and family history

In the control group of 72 patients $28(38.9 \%)$ were men and $44(61.1 \%)$ were women, with a man: woman ratio of 0.63 . The average age at diagnosis for men and women was nearly the same $(63.4$ years 
and 65.4 years). The median age was separated by 3 years: men, 64 years; women, 67 years.

Nine patients $(12.5 \%)$ gave a family history of glaucoma.

\section{Refractive error}

Hyperopia was found in $45.9 \%, 1$ eye as great as $+7.00 \mathrm{D}$ spherical equivalent. Myopia (greater than $-1.00 \mathrm{D}$ spherical equivalent) was present in only $14.0 \%$, with 1 patient having myopia of $-6.00 \mathrm{D}$ spherical equivalent. Emmetropia $(-0.75$ to $+0.75 \mathrm{D}$ spherical equivalent) was found in $43 \%$.

\section{Associated ophthalmic abnormalities}

No eyes suffered retinal detachment. One patient had bilateral congenital cataracts, 1 had bilateral choroidal sclerosis, and 1 had bilateral macular degeneration. Occlusion of the central retinal vein occurred in 1 eye.

\section{RESPONSE TO THERAPY}

\section{Medical therapy}

The majority of eyes (122 eyes, $85.4 \%$ ) with chronic simple glaucoma were controlled medically. The pressure was controlled by pilocarpine 1 to $4 \%$ in 94 eyes $(77.0 \%)$. Epinephrine alone was effective in 11 eyes $(9.0 \%)$ and was used as an adjunct to pilocarpine in 7 eyes $(5.7 \%)$ more. Other types of miotics were used in $10(8.3 \%)$ of the eyes.

In the group with pigment dispersion and increased pressure pilocarpine alone was used in 38 eyes $(40 \cdot 4 \%)$. Epinephrine alone was used in 36 eyes $(38.3 \%)$ and in combination with pilocarpine in 15 eyes $(16.0 \%)$. Other types of therapy were used in 2 eyes $(2 \cdot 1 \%)$, and no treatment was used in 3 eyes $(3.2 \%)$.

$76 \%$ of the 204 eyes with pigmentary glaucoma were controlled by medical therapy. Pilocarpine, 1 to $4 \%$, was successful in 65 eyes $(31.9 \%)$. Epinephrine and pilocarpine used in conjunction were successful in 42 eyes $(20.6 \%)$, and epinephrine alone in 34 eyes $(16.7 \%)$. Other miotics were used in 14 eyes $(6 \cdot 8 \%)$.

\section{Surgical therapy}

A filtering operation was done when medical therapy failed.

(1) Chronic simple glaucoma. An operation was necessary in $21(14.6 \%)$ eyes of 13 patients. Eight of the patients ( 2 men, 6 women) had bilateral surgery. The average age at surgery was 65.8 (4 men) and 62.9 ( 9 women). The age range at the time of surgery was 58 to 78 years. None of the eyes required more than 1 operative procedure.

(2) Pigmentary glaucoma. Filtering operations were done on $48(23.5 \%)$ eyes of 32 patients. Two- thirds of the patients (21) were men. They came to surgery at an average age 11 years younger than the women (men, 40.8 years; women, $51 \cdot 1$ years). The youngest pigmentary glaucoma patient requiring surgery was 21 years old and oldest, 72 years. Eight eyes required more than one surgical procedure.

\section{Discussion}

The true incidence of pigment dispersion syndrome is difficult to ascertain, especially in the older literature. Krukenberg spindles can easily be overlooked on routine examination unless careful slitlamp biomicroscopy and gonioscopy are done. Only 353 patients with pigment dispersion syndrome in series of 15 or more patients each have been described in the English literature. ${ }^{3-7}$ The 202 patients reported by Evans et al. ${ }^{8}$ were not included because the report included numerous eyes that had suffered trauma and inflammation. Sugar and Barbour ${ }^{9}$ originally reported the association of glaucoma and pigment dispersion in 2 patients. In a subsequent report Sugar ${ }^{3}$ reported 17 of his own patients and 111 from the literature having pigmentary glaucoma.

The 407 patients ( 799 eyes) with pigment dispersion syndrome reported in this paper were gathered over a period of approximately 30 years from the private practice of one of us (H.G.S.) and were part of a series of 9200 glaucoma patients of various types seen during the same period. They comprised $4.4 \%$ of that group. An average of 13.1 patients per year was seen with pigment dispersion syndrome and an average of 3.3 patients was seen with pigmentary glaucoma. The incidence in the general population obviously is much smaller than in a population of glaucoma patients.

The present study confirms earlier reports that pigment dispersion syndrome with associated glaucoma is more frequent in men than in women by a ratio of approximately $3: 2$. The onset of glaucoma occurs at an earlier age in men than in women. The dispersion syndrome itself, even without glaucoma, is present at a younger age in men than in women. The sex incidence in our control group of patients with chronic simple glaucoma was nearly the reverse of that found in pigmentary glaucoma, the ratio being $2: 3$ men to women. The average age for men and women in chronic simple glaucoma was nearly alike, being 63.4 years for men and 65.4 years for women. A hormonal factor might be suggested to explain such differences in sex incidence and age of onset.

A positive family history for glaucoma was elicited in $4 \%$ of our patients with pigment dispersion syndrome, $16 \%$ with pigmentary glaucoma, and $12.5 \%$ with chronic simple glaucoma. Hereditary 
factors undoubtedly play a part in the aetiology of both pigmentary and chronic simple glaucoma. The low incidence suggests a multifactorial inheritance pattern or trait of variable penetrance and expressivity.

In this present study and in an earlier study iris colour had no relationship to pigmentary glaucoma or to the control group. The iris stroma in pigment dispersion syndrome and pigmentary glaucoma characteristically has a normal appearance, although pigment flecks may be seen on the surface of the iris. A characteristic finding in pigment dispersion syndrome, with or without glaucoma, is transillumination of the iris near its periphery, which results from atrophy of the pigment epithelial layers of the iris. The atrophy may be caused by congenital ${ }^{10}$ or mechanical factors. ${ }^{11}$ Transillumination is undoubtedly noted more frequently in blue irides than in brown because light is transmitted more freely through a lightly pigmented blue iris than a brown. Iris epithelial atrophy must therefore be more advanced in the brown than in the blue iris to be demonstrable.

Gonioscopy shows characteristic heavy pigmentation over the entire circumference of the posterior half of the trabeculum overlying the canal of Schlemm. In some eyes the pigment deposition may cover the entire width of the trabeculum and even involve the periphery of the cornea adjacent to Schwalbe's line. Although Lichter $^{12}$ reported an increased incidence of iris processes in patients with pigmentary glaucoma, our observations did not support this claim. Almost all of the eyes with pigment dispersion syndrome and pigmentary glaucoma had grade $\mathbf{I I I}^{2}$ or greater angle pigmentation.

In those patients with pigmentary glaucoma where the glaucoma was more severe or advanced in one eye than in the other the eye with the higher pressure and more advanced signs of glaucoma invariably had the more heavily pigmented anterior chamber angle. In those patients the more heavily pigmented eye also was more resistant to treatment and more likely to require surgical intervention.

This observation offers quite convincing evidence that pigment is a very important aetiological factor in pigmentary glaucoma. The fact that glaucoma is not always associated, even in the heavily pigmented angles, could be explained by assuming an individual susceptibility of trabecular meshwork to obstruction of aqueous outflow. The histological and ultrastructural studies by Rodrigues et al. ${ }^{13}$ indicated that unique changes occurred in the juxtacanalicular meshwork in association with excessive amounts of pigmented epithelial melanosomes. Richardson et $a l .^{14}$ found ultrastructural changes in the trabecular endothelium and trabecular sheets thought to be initiated by the presence of increased amounts of melanin pigment.

The fact that grade IV pigmentation of the anterior chamber angle is not always associated with a physiologically significant increase in intraocular pressure indicates that some factor in addition to the amount of pigment must be involved in the mechanism of pigmentary glaucoma. A hormonal element might be suggested because the condition occurs earlier and in greater incidence in men than in women. Pore size of the trabecular meshwork is another possible factor. Aqueous flow in an eye with smaller trabecular pores might be more easily obstructed than in an eye with larger pores. These physiological diameters may be difficult to demonstrate in formalin fixed eyes.

The association of myopia with pigmentary glaucoma and pigment dispersion syndrome is well known. The distribution of refractive error in young male military recruits was studied by Sorsby et al. ${ }^{15}$ It was found that $6.6 \%$ of the patients had myopic refractive errors of $-1.00 \mathrm{D}$ spherical equivalent or greater, $45.1 \%$ were between $-0.9 \mathrm{D}$ and $+0.90 \mathrm{D}$ spherical equivalent, and $48.3 \%$ had refractive errors of $+1.00 \mathrm{D}$ spherical equivalent or greater. Against these criteria all studies of pigmentary dispersion with or without glaucoma show a much higher incidence of myopia than in Sorsby et al.'s study. On the other hand the incidence of refractive error in our chronic simple glaucoma group differed little from these workers' findings.

The high incidence of retinal detachment $(6.6 \%$ in normotensive group and $7.6 \%$ in glaucoma group) has not been mentioned previously. Phakic, non-traumatic retinal detachment has been reported at an annual incidence of approximately $0.01 \%$ to $0.005 \%$ in the general population. The majority of retinal detachments in our study were found in men who were phakic but not highly myopic. The degree of increased risk for retinal detachment with pigment dispersion syndrome seems substantial.

Epinephrine alone or in combination with pilocarpine appears to be more effective in lowering the intraocular pressure in pigmentary glaucoma than in chronic simple glaucoma. However, this drug was not studied in a controlled prospective manner. Although Sugar ${ }^{3}$ noted that pigmentary glaucoma was more easily controlled in its early stages, he did not outline his treatment. Bick ${ }^{16}$ stated that it was more difficult to control the increased intraocular pressure of women than of men.

In our study medical therapy was somewhat more effective in controlling the pressure in the chronic simple glaucoma group (85\%) than in the pigmentary glaucoma group $(66 \%)$. The average age of 
patients with pigmentary glaucoma requiring surgery was nearly 15 years younger than those patients with chronic simple glaucoma requiring surgery. Many more patients with pigmentary glaucoma required multiple surgical procedures to attain satisfactory pressure control.

\section{References}

1 Scheie HG, Fleischhauer HW. Idiopathic atrophy of the epithelial layers of the iris and ciliary body. Arch Ophthalmol 1958; 59: 216-88.

2 Scheie HG. Width and pigmentation of the angle of the anterior chamber. A system of grading by gonioscopy. Arch Ophthalmol 1957; 58: 510-2.

3 Sugar HS. Pigmentary glaucoma: a 25-year review. Am J Ophthalmol 1966; 62: 499-507.

4 Lichter PR, Schaffer RN. Diagnostic and prognostic signs in pigmentary glaucoma. Trans Am Acad Ophthalmol Otolaryngol 1970; 74: 984-98.

5 Wilenski JT, Buerk KM, Podos SM. Krukenberg spindles. Am J Ophthalmol 1975; 79: 220-5.

6 Zink HA, Palmberg PF, Sugar A, et al. Comparison of in vitro corticosteroid response in pigmentary glaucoma and primary open-angle glaucoma. Am J Ophthalmol 1975; 80: 478-84.
7 Weingeist TA. The pigment dispersion syndrome and glaucoma. Ophthalmic Semin 1976; 1: 371-412.

8 Evans WH, Odom RE, Wenaas EJ. Krukenberg's spindle. A study of two hundred and two collected cases. Arch Ophthalmol 1941; 26: 1023-56.

9 Sugar HS, Barbour FA. Pigmentary glaucoma. A rare clinical entity. Am J Ophthalmol 1949; 32: 90-2.

10 Fine BS, Yanoff M, Scheie HG. Pigmentary 'glaucoma', a dysplasia of the iris. Trans Am Acad Ophthalmol Otolaryngol 1974; 78: 314-25.

11 Campbell DG. Pigmentary dispersion and glaucoma. A new theory. Arch Ophthalmol 1979; 97: 1667-72.

12 Lichter PR. Pigmentary glaucoma-current concepts. Trans Am Acad Ophthalmol Otolaryngol 1974; 78: OP309-13.

13 Rodrigues MM, Spaeth GL, Winreb S, Sivalingham E. Spectrum of trabecular pigmentation in open angle glaucoma. Trans Am Acad Ophthalmol Otolaryngol 1976; 81: OP 258-76.

14 Richardson TM, Hutchinson BT. Grant MW. The outflow tract in pigmentary glaucoma: a light and electronmicroscopic study. Arch Ophthalmol 1977; 95: 1015-25.

15 Sorsby A, Sheridan M, Leary RA, Benjamin B. Vision, visual acuity, and ocular refraction of young men. $B r$ Med J 1960; i: 1394-8.

16 Bick MW. Pigmentary glaucoma in females. Arch Ophthalmol 1957; 58: 483-94. 